\title{
Integration of Fault Notification Mechanism for IoT-based Photovoltaic \\ Panel Monitoring and Analysis System
}

\section{DOI : 10.36909/jer.14243}

Muhammad Faiz Aqil bin Ahmad Fairuz, Ranjit Singh Sarban Singh*, Soo Yew Guan

Advanced Sensor and Embedded Control (ASECs) Research Group, Centre for Telecommunication Research \& Innovation (CeTRI), Fakulti Kejuruteraan Elektronik dan Kejuruteraan Komputer (FKEKK), Universiti Teknikal Malaysia Melaka (UTeM), Hang Tuah Jaya, 76100, Durian Tunggal, Melaka, Malaysia.

*Email: ranjit.singh@utem.edu.my; Corresponding Author.

\begin{abstract}
The necessity of fault detection and notification has been exploited by the growth of renewable energy systems. The application of fault detection and notification is seen as an effective tool to monitor the performance of renewable energy systems. Looking at the importance of fault detection and notification integration into renewable energy systems based on the conducted literature review, this paper presents fault notification mechanism and implementation, which also consist of detection in an IoT-based photovoltaic panel monitoring and analysis system. The implemented fault notification detects the abnormalities of the voltage-currenttemperature in the IoT-based photovoltaic panel monitoring and analysis system. The implemented fault notification in the IoT-based photovoltaic panel monitoring and analysis detects and notify any sort of abnormality of the voltage-current-temperature that is recorded at the photovoltaic panel. The implemented fault notification operates based on the threshold values of the voltage-current-temperature that has been programmed in the centralised controller. Hence, when any parameter input is below the preset threshold value, an email notification is sent to the centralised system to notify the maintenance team. Based on the
\end{abstract}


implementation and obtained results, the implemented fault notification in the developed IoTbased photovoltaic panel monitoring and analysis system successfully performed and validated the purpose of the implementation.

Keywords: fault detection and notification system, solar health monitoring system, internet of things fault notification, solar health photovoltaic system.

\section{INTRODUCTION}

As the world accelerates in technological development, many developments have been designed and developed to provide an output for user access. To mention precisely, today's developed products or systems are self-regulated, especially in decision-making in terms of detecting faults (M. H. Hwang et al., 2021). Having said about self-regulated and decision making, one of the mechanisms that are very important in these kinds of products or systems is the fault detection and notification mechanism (Srinivas et al., 2021), (Abbas \& Zhang, 2021). Fault detection and notification are an important mechanism in self-regulated or decision-making products or systems to update the products or systems behavior, condition, performance, and many other criteria (Sepehrirad et al., 2020). Having briefly mentioned the purpose of integrating the fault detection and notification mechanism in products or systems, this paper introduces fault notification mechanism into the developed IoT-based photovoltaic panel monitoring and analysis system. The integration of fault notification mechanism is to continuously assist the developed IoT-based photovoltaic panel monitoring and analysis system in checking on the voltage-current-temperature parameters. To further explain the integrated fault notification mechanism IoT- based photovoltaic panel monitoring and analysis system, this paper has been arranged as follows.

The following section explains the literature review of the available systems or systems that have been developed to detect the fault of the solar systems and the notification mechanism used to update the system's fault. Then, a brief methodology of the proposed fault notification 
mechanism shown in Figure 1 operation and functionality is explained. Results of the fault notification that have been captured when the IoT-based Photovoltaic Panel Monitoring and Analysis System is operating is presented in Section IV, and finally, this research has been concluded.

\section{LITERATURE REVIEW}

As the previously developed systems are studied, the studies show that the developed systems are used to measure, record, monitor, and analyze the developed system's performances. As the requirement to measure, record, monitor and analyze the developed system's performances are important, it is also seen that it is important for a system to provide some sort of notification when an abnormality occurs during the system's operation. Hence, this section provides a short review of the types of available fault detection and notification is studied, and an appropriate method of fault notification is proposed to be adopted into the proposed IoT-based Photovoltaic Panel Monitoring and Analysis System. With that, in (Lazzaretti et al., 2020) recursive linear model has been introduced to detect faults in the proposed system. The recursive linear model uses the solar photovoltaic panel's irradiance and temperature as input signals and the solar photovoltaic panel's power as an output parameter. Besides that, the machine learning method has been adopted to classify the faults such as short-circuit, opencircuit, partial shadowing, and degradation. By integrating the fault detection and classification in this proposed research, it allows assisting the power system to be monitored online and in real-time. In another different research presented by (Rico Espinosa et al., 2020), this research focuses on identifying automatic physical fault classification for photovoltaic plants using the convolutional neural networks. The faults have been classified based on the RGB image, semantic segmentation to extract the features of the photovoltaic panel to classify the type of available fault is identified. In another research by (H. R. Hwang et al., 2019), open-circuit voltage is obtained using the neural network method to detect the error rate between the initial measured data and actual measured data. This method detects the immediately preceding value 
during the system operational, and this immediately preceding value is detected as a possible or potential occurring fault.

Synchronized Thermography (ST) is another method used to detect the photovoltaic panels faults in the outdoor environment. The ST technique has been presented by (Schuss et al., 2020) in their research and the analysis shows that illustrating the captured images under changing irradiation and different angles assists rapid defects detections of a photovoltaic panel. Also, this technique reported that the maintenance team provides quick responds to the defect repair process.

In another different research (Adhya et al., 2016), it is mentioned that the cost of renewable energy equipment is reducing with the technology advancement, which encourages massive scale of solar photovoltaic installations. Among the fastest-growing technologies, IoT seems to lead quicker and smarter. The main idea in this (Adhya et al., 2016) research is that each solar photovoltaic panel should be monitored to know the solar photovoltaic panel's current status. The monitoring is important for the solar photovoltaic panel's performance evaluation and directly provides analysis of the solar photovoltaic panel's condition. Therefore, the performance, monitoring, and maintenance of the solar photovoltaic plant can be enhanced by integrating the IoT-based technology. The IoT technology also can be significantly enhanced to facilitate preventive maintenance, fault detection, analysis of the plant as well as real-time plant observations. For example, if there is decreased light intensity, solar photovoltaic panels automatically change their direction to absorb maximum light intensity so that the solar photovoltaic panel can efficiently perform energy conversion.

Besides that, the development of innovative fault detection and diagnosis scheme which assists in monitoring the direct current (DC) of the solar photovoltaic system seems to be necessary. Research conducted by (Harrou et al., 2018) suggested a statistical approach that exploits the advantages of one-diode model, and those of the Univariate Exponentially Weighted Moving Average (UEWMA) and Multivariate Exponentially Weighted Moving Average (MEWMA) 
charts are better used to detect the types of faults. Specifically, the generated residual of current, voltage, and power is predicted using the Maximum Power Point Tracking (MPPT). With that, the Multivariate Exponentially Weighted Moving Average (MEWMA) monitoring chart is adopted to detect the residual faults. Even though the residual faults can be detected, the Multivariate Exponentially Weighted Moving Average (MEWMA) scheme is cannot specifically identify the type of fault. Therefore, once the fault is detected, the Univariate Exponentially Weighted Moving Average (UEWMA) chart, which is based on current and voltage indicators, is used to identify the type of fault that occurs, such as short-circuit, opencircuit and shading faults.

Other than that, there are also many difficulties in extending the application of solar photovoltaic systems as an electricity generation system, one of which is to timely detect the fault to preserve the efficiency and high productivity of the solar photovoltaic systems. In a newly presented research by (Natsheh \& Samara, 2020), a new and efficient algorithm which is for fault diagnosis based on an artificial intelligent Nonlinear Autoregressive Exogenous (NARX) neural network and Sugeno fuzzy inference is proposed to isolate and identify the faults that may occur in a solar photovoltaic system. The fuzzy inference also includes the real sensed output power from the solar photovoltaic system as well as able to sense the surrounding condition.

Next, to obtain the solar photovoltaic device output capacity forecast, an artificial intelligent Nonlinear Autoregressive Exogenous (NARX) based on a neural network is used. The NARX helps to actual obtained the solar photovoltaic system's real output power and the ambient conditions, which is via the connected sensors. The developed algorithm is implementable on a low-cost microcontroller. It performs to detect several faults causes as open and short circuit degradation, detective Maximum Power Point Tracking (MPPT) and partial shading (PS). In addition to the fault detection, the algorithm is also to capture the radiation and temperature, which is used to decide the point of the maximum power of the solar photovoltaic system. 
An advanced sensor for measuring the real-time operating voltage and current for open-circuit voltage and short circuit current for the string connected of solar photovoltaic panels has been developed by (Guerriero et al., 2016). To efficiently conduct the measurement of the voltage and current, the disconnection of the measuring device, especially the sensor should not influence the actual behavior of the string operational. Next, the sensor does not require additional cables to install the wireless communication and energy harvesting system to supply the power supply for the developed system. Moreover, the developed system also demonstrated the reliability and usefulness of the sensor to continuously monitor the voltage and current of the solar photovoltaic panels in a plant. Also, the system has been developed with the ability to detect the faults in a solar photovoltaic string and able to correctly localize the malfunctioning panels in the solar photovoltaic plant.

Based on the conducted study about fault detection and notification, it is found that developed faults detection technique is mostly using the soft computing method, which is like image processing, neural network, and convolutional neural networks.

The study of fault detection and notification also explains the importance of conducting the solar photovoltaic panel monitoring to have consistent output current and voltage while the solar photovoltaic panels are operating.

Therefore, this finding has been used to understand the available techniques and develop the proposed fault notification for the IoT-based Photovoltaic Panel Monitoring and Analysis System. The fault notification mechanism proposed in this research has been integrated into our previously developed system (Fairuz et al., 2019).

\section{METHODOLOGY}

Figure 1 shows the operational process of the integrated current-voltage-temperature sensors and fault notification operation at the system. At the beginning of the operation, the INA 219 DC Current/Voltage and Thermocouple Amplifier MAX31855 Sensors are initialized to check the sensors connectivity for the sensing task. 
In the second stage, there are two operating scenarios, first is the INA 219 DC Current/Voltage and Thermocouple Amplifier MAX31855 (TC1, TC2, TC3, and TC4) sensors starts to sense and measure the parameter of current, voltage, and temperature of the solar photovoltaic panel. If the read parameters are more or equal to the threshold value, then the measured current, voltage and temperature values are transferred into the Raspberry Pi Zero Wireless SD-Card Storage System. These stored values are then wirelessly sent over into the .php database at the localhost computer database. In the second scenario, if any of the read current, voltage or temperature value is below the pre-set threshold, then a fault notification is updated to the maintenance team via email, as shown in Figure 3 and Figure 5 in Section IV. The fault notification email contains information such as solar photovoltaic panel identification and sensor number identification. This two information is required by the maintenance team to attend to perform the fault repair immediately.

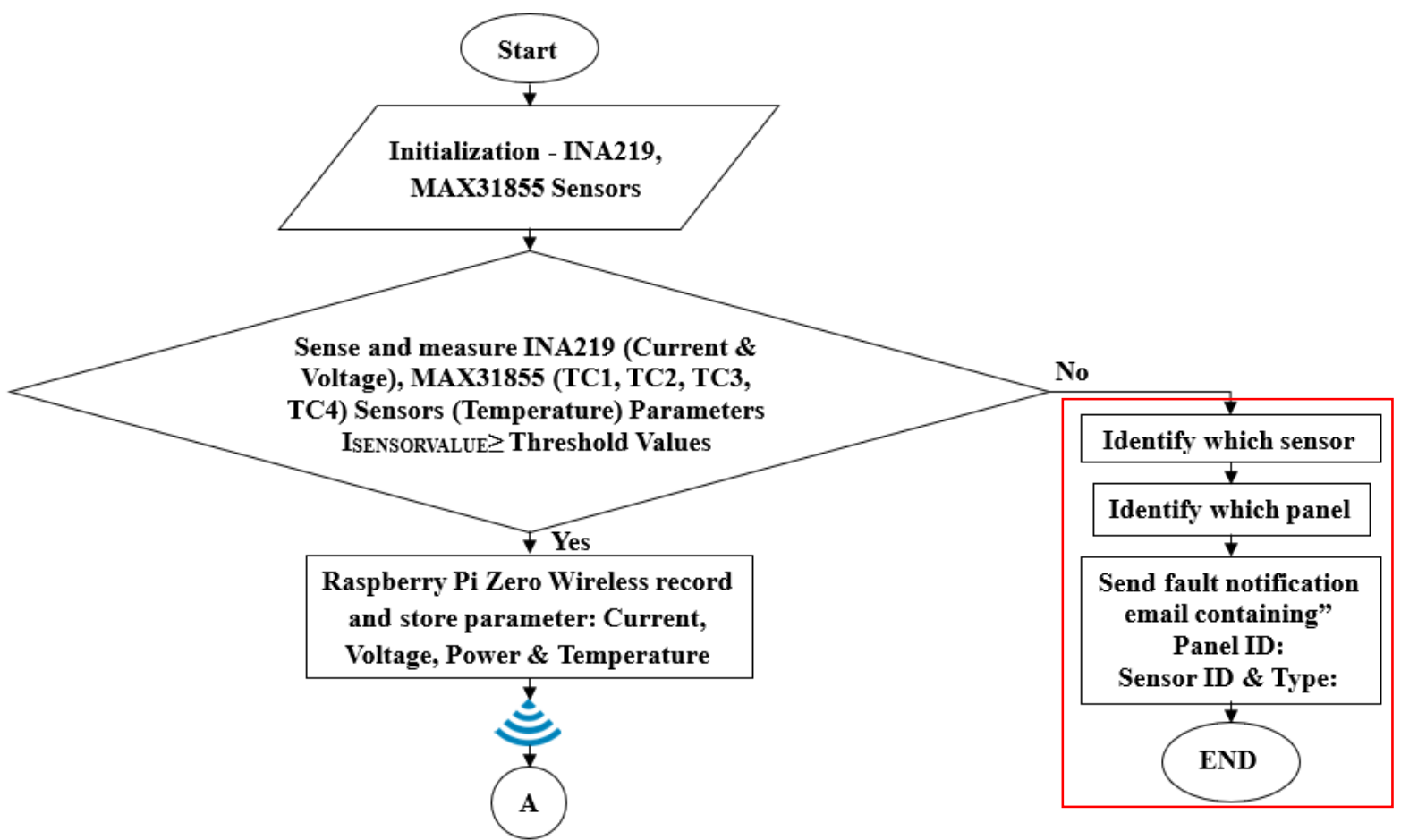

Figure 1 Process of current-voltage and temperature sensors and fault notification operation 


\section{RESULTS AND DISCUSSION}

This section explains the captured email results of the fault notification implemented into the Hardware Design Platform for IoT-based Photovoltaic Monitoring and Analysis System, which detects the current and temperature abnormality when the system is operating. When a current abnormality is detected, the current value is recorded into the Raspberry Pi Zero Wireless SD-Card Storage System as shown in Figure 2.

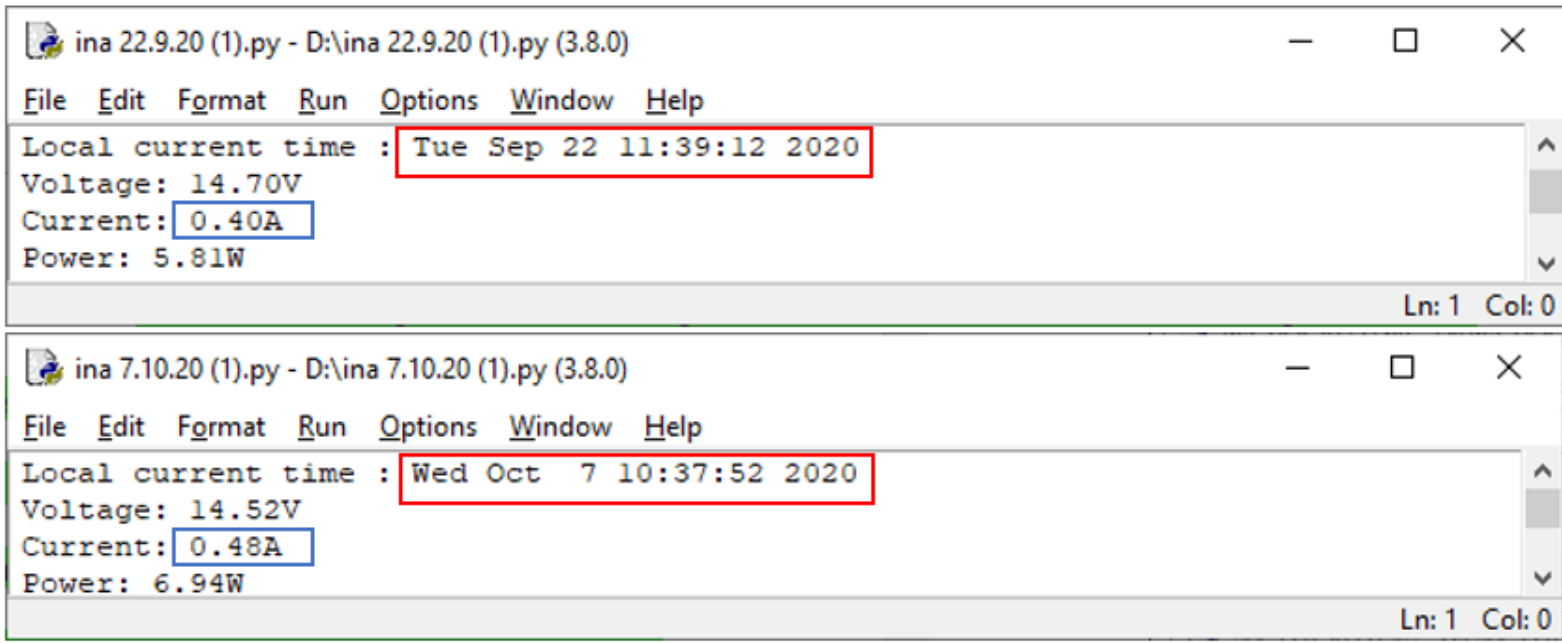

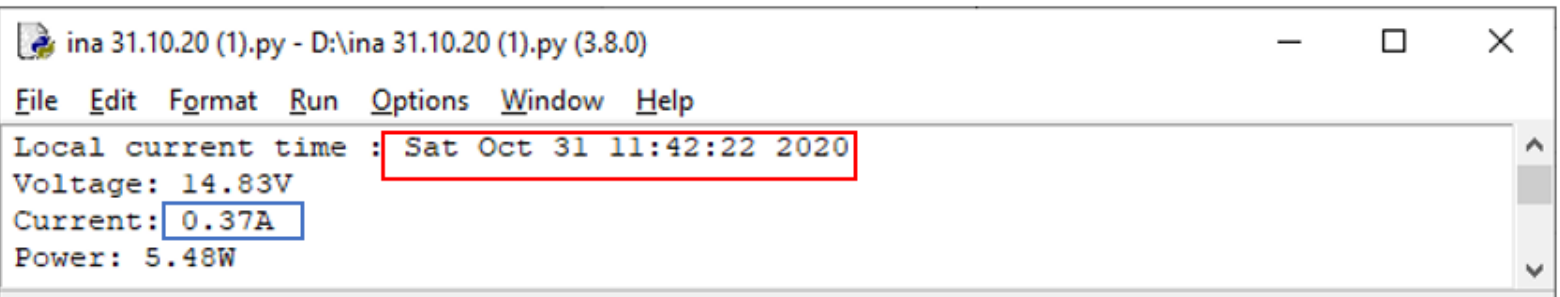

Time Fault extracted information $\quad \square$ Fault notification email

Figure 2 Current fault notification - INA219 DC current/voltage sensor - Raspberry Pi Zero Wireless SD-Card system

At the same, simultaneously fault notification email, as shown in Figure 3, is sent to the registered email to inform the maintenance team of the detected faults, as shown in Figure 2. Based on Figure 2, there are three faults detected during the system operation and functionality which is also informed in Figure 3. The abnormalities that are captured in Figure 3 is related to the sensed and measured current at the INA219 DC Current/Voltage Sensor at different 
operating time.

The captured results, shown in Figure 3, also provide the details of the sensor, such as the solar photovoltaic panel identification detail (ID), Sensor ID, and Fault Type, as shown in Figure 1.

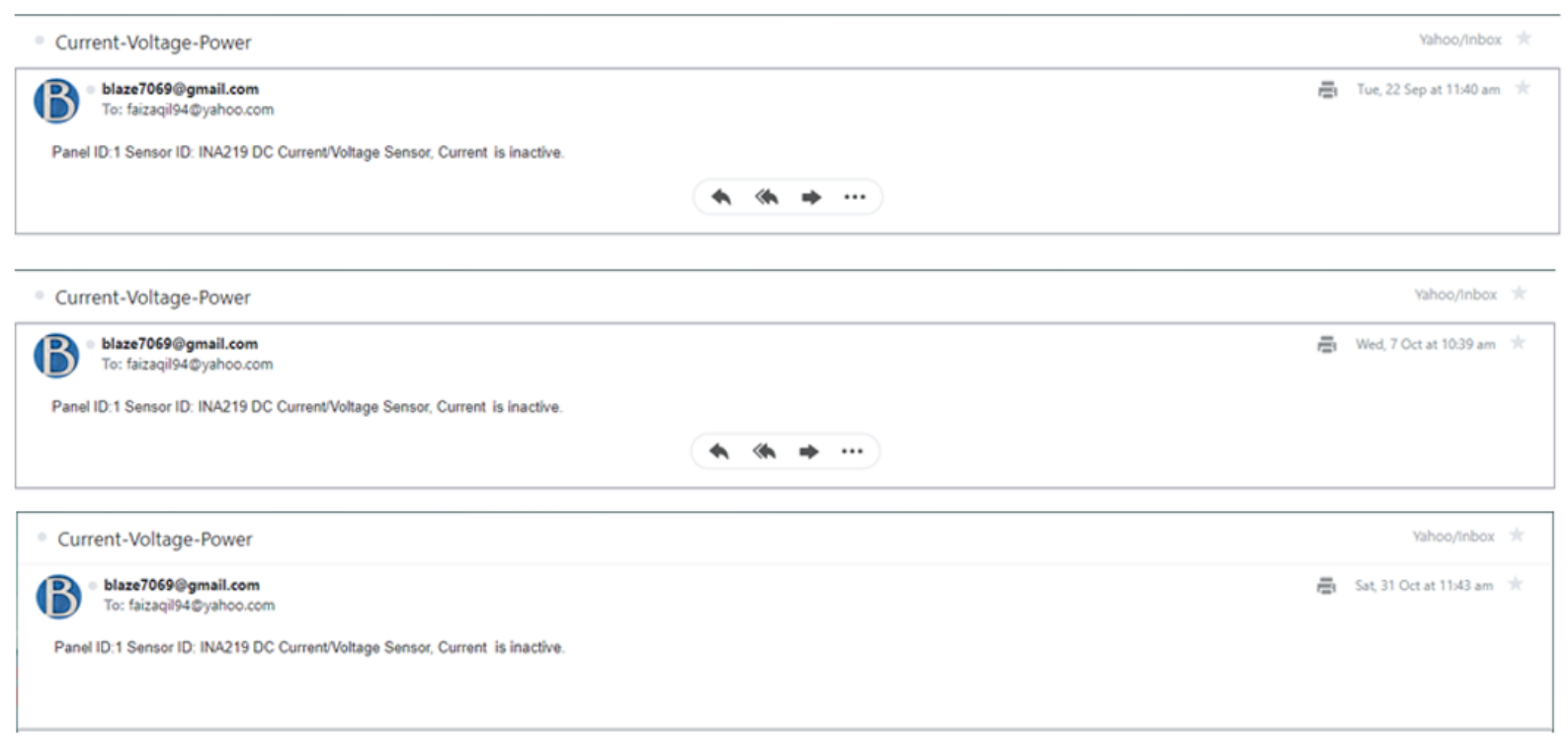

Figure 3 Fault notification email INA219 DC current/voltage sensors - current (A)

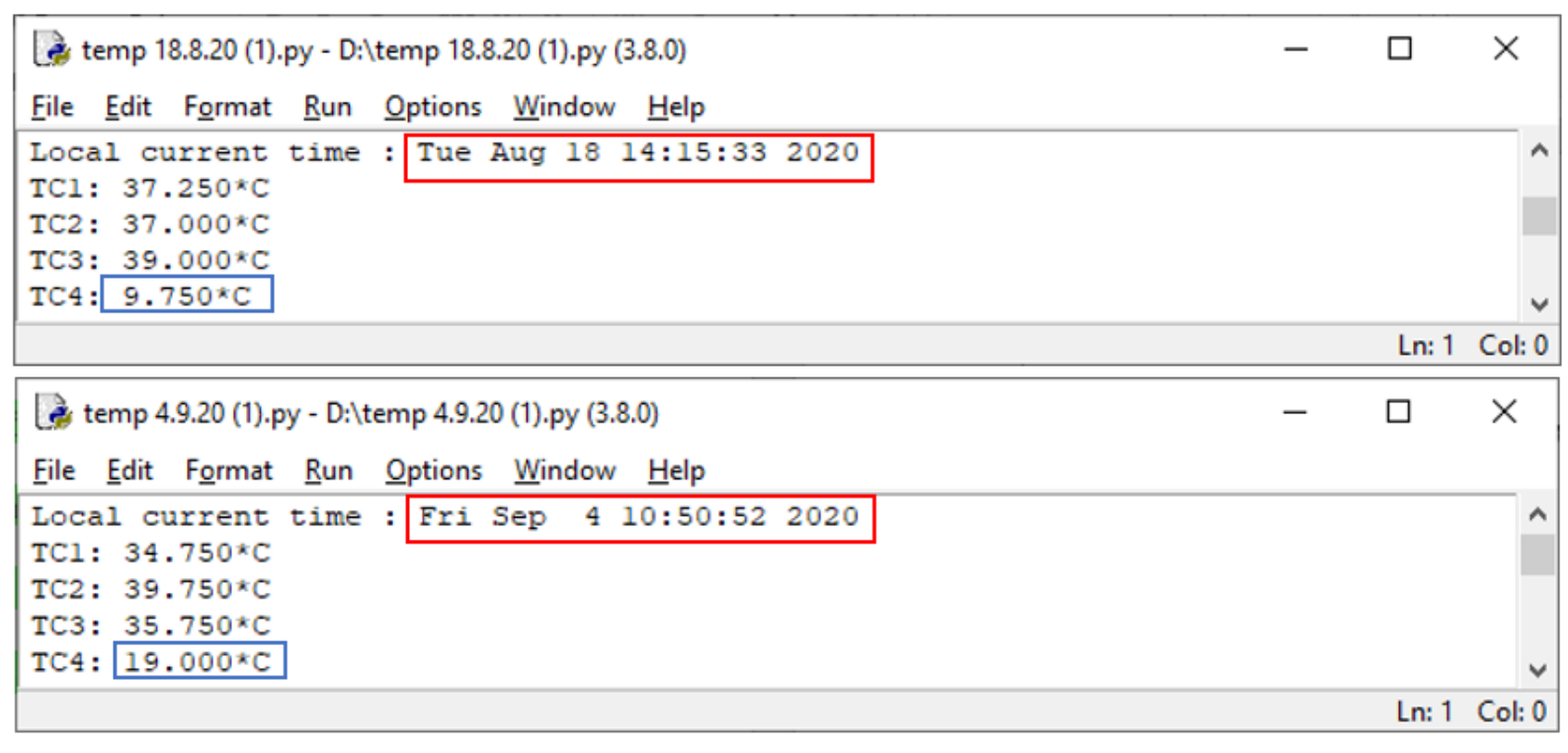

\section{$\square$ Time Fault extracted information $\quad \square$ Fault notification email}

Figure 4 Fault notification temperature (TC4) - sensed and measured four (4) temperature sensors - Thermocouple Amplifier MAX31855 Sensor - Raspberry Pi Zero Wireless SD-Card system 
Figure 5 Fault notification email Thermocouple Amplifier MAX31855 Sensors - TC4

Next, this section explains the captured email results of the fault notification that is implemented into the Hardware Design Platform for IoT based Photovoltaic Monitoring and Analysis System which detects any kind of temperature abnormality that is sensed and measured via the Thermocouple Amplifier MAX31855 Sensor integrated with the Raspberry Pi Zero Wireless microcontroller. When a temperature abnormality is detected, the temperature value is recorded into the Raspberry Pi Zero Wireless SD-Card Storage System, as shown in Figure 4. At the same, simultaneously fault notification email, as shown in Figure 5 , is sent to the registered email to inform the maintenance team of the detected fault. Based on Figure 5, the fault notification shows two different temperature abnormalities are detected as fault during the system operation and functionality, which is also shown in Figure 5. The abnormalities captured in Figure 5 are related to the sensed and measured temperature at the Thermocouple Amplifier MAX31855 Sensor at different operating periods. The captured results shown in Figure 5 also provide the details of the sensor, such as the solar photovoltaic panel identification detail (ID), Thermocouple Amplifier MAX31855 Sensor ID, and Fault Sensor. 


\section{CONCLUSION}

This section concludes the outcome of the proposed fault notification that has been integrated into the IoT-based Photovoltaic Panel Monitoring and Analysis System. The proposed methodology can successfully detect any abnormality occurs in the IoT-based Photovoltaic Panel Monitoring and Analysis System during the operation. The presented results also shows that the detected faults are successfully notified to the maintenance team for quick response and repair. Also, some configurations such as providing the panel identification detail (ID), Thermocouple Amplifier MAX31855 Sensor ID, and Fault Sensor must be manually configured by the system programmer. The developed fault notification is unable to selfprogram this information when the system starts to operate.

\section{ACKNOWLEDGEMENT}

The author(s) wish to acknowledge the support from the Ministry of Higher Education of Malaysia (MOHE), Centre for Telecommunication Research \& Innovation (CeTRI), Faculty of Electronic and Computer Engineering (FKEKK), Universiti Teknikal Malaysia Melaka (UTeM), Hang Tuah Jaya, 76100, Durian Tunggal, Melaka, Malaysia.

\section{REFERENCES}

Abbas, M., \& Zhang, D. (2021). A smart fault detection approach for PV modules using Adaptive Neuro-Fuzzy Inference framework. Energy Reports, 7, 2962-2975. https://doi.org/10.1016/j.egyr.2021.04.059

Adhya, S., Saha, D., Das, A., Jana, J., \& Saha, H. (2016). An IoT based smart solar photovoltaic remote monitoring and control unit. 2016 2nd International Conference on Control, Instrumentation, Energy and Communication, CIEC 2016, January, 432-436. https://doi.org/10.1109/CIEC.2016.7513793

Fairuz, M. F. A. A., Singh, R. S. S., \& Nurdin, M. I. (2019). Methodological hardware design and development of health monitoring. Proceeding of Mechnical Engineering Research 
Day 2019, October, 205-206.

Guerriero, P., Di Napoli, F., Vallone, G., Dalessandro, V., \& Daliento, S. (2016). Monitoring and diagnostics of PV plants by a wireless self-powered sensor for individual panels. IEEE Journal of Photovoltaics, 6(1), 286-294. https://doi.org/10.1109/JPHOTOV.2015.2484961

Harrou, F., Sun, Y., Taghezouit, B., Saidi, A., \& Hamlati, M. E. (2018). Reliable fault detection and diagnosis of photovoltaic systems based on statistical monitoring approaches. Renewable Energy, 116, 22-37. https://doi.org/10.1016/j.renene.2017.09.048

Hwang, H. R., Kim, B. S., Cho, T. H., \& Lee, I. S. (2019). Implementation of a Fault Diagnosis System Using Neural Networks for Solar Panel. International Journal of Control, Automation and Systems, 17(X), 1-9. https://doi.org/10.1007/s12555-018-01533

Hwang, M. H., Kim, Y. G., Lee, H. S., Kim, Y. D., \& Cha, H. R. (2021). A study on the improvement of efficiency by detection solar module faults in deteriorated photovoltaic power plants. Applied Sciences (Switzerland), 11(2), 1-16. https://doi.org/10.3390/app11020727

Lazzaretti, A. E., da Costa, C. H., Rodrigues, M. P., Yamada, G. D., Lexinoski, G., Moritz, G. L., Oroski, E., de Goes, R. E., Linhares, R. R., Stadzisz, P. C., Omori, J. S., \& Dos Santos, R. B. (2020). A monitoring system for online fault detection and classification in photovoltaic plants. Sensors (Switzerland), 20(17), 1-30. https://doi.org/10.3390/s20174688

Natsheh, E., \& Samara, S. (2020). Tree search fuzzy narx neural network fault detection technique for PV systems with IOT support. Electronics (Switzerland), 9(7), 1-26. https://doi.org/10.3390/electronics9071087

Rico Espinosa, A., Bressan, M., \& Giraldo, L. F. (2020). Failure signature classification in solar 
photovoltaic plants using RGB images and convolutional neural networks. Renewable Energy, 162(August), 249-256. https://doi.org/10.1016/j.renene.2020.07.154

Schuss, C., Remes, K., Leppanen, K., Saarela, J., Fabritius, T., Eichberger, B., \& Rahkonen, T. (2020). Rapid fault diagnosis of photovoltaic panels under outdoor environmental conditions. I2MTC 2020 - International Instrumentation and Measurement Technology Conference, $\quad$ Proceedings, $\quad$ 1-6. https://doi.org/10.1109/I2MTC43012.2020.9129309

Sepehrirad, I., Ebrahimi, R., Alibeiki, E., \& Ranjbar, S. (2020). Intelligent differential protection ccheme for controlled islanding of microgrids based on decision tree tTechnique. Journal of Control, Automation and Electrical Systems, 31(5), 1233-1250. https://doi.org/10.1007/s40313-020-00588-7

Srinivas, S., Anand, D. D. G., \& Chandrakanth, D. H. G. (2021). Improving the efficiency of a photovoltaic module by fault diagnosing, cleaning \& dual axis solar tracking using internet of things ( IOT). Proceedings of the First International Conference on Computing, Communication and Control System, I3CAC 2021. https://doi.org/10.4108/eai.7-62021.2308613 